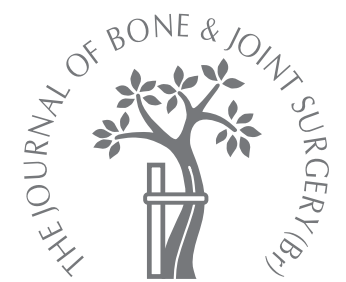

- REVIEW ARTICLE

\title{
Outcome measures for orthopaedic interventions on the hip
}

E. Ashby,

M. P. W. Grocott, F. S. Haddad

\section{From University}

College London

Hospital, London, England

E. Ashby, Orthopaedic Research Clinical Fellow F. S. Haddad, Consultant Orthopaedic Surgeon University College London Hospital, 235 Euston Road, London NW1 2BU, UK.

M. P. W. Grocott, ITU Consultant Surgeon Whittington Hospital NHS Trust, Magdala Avenue, London N19 5HF, UK.

Correspondence should be sent to Miss E. Ashby at Sky Ward, Great Ormond Street Hospital for Children NHS Trust, Great Ormond Street, London WC1N 3JH,UK; e-mail: elizabethashby@doctors.org.uk

(C)2008 British Editorial Society of Bone and Joint Surgery doi:10.1302/0301-620X.90B5. $19746 \$ 2.00$

$J$ Bone Joint Surg [Br] 2008;90-B:545-9.

\begin{abstract}
Orthopaedic outcome measures are used to evaluate the effect of operative interventions. They are used for audit and research. Knowledge of these measures is becoming increasingly important with league tables comparing surgeons and hospitals being made accessible to the profession and the general public.

Several types of tool are available to describe outcome after hip surgery such as generic quality-of-life questionnaires, disease-specific quality-of-life questionnaires, hip-specific outcome measures and general short-term clinical measures. We provide an overview of the outcome measures commonly used to evaluate hip interventions.
\end{abstract}

Orthopaedic interventions are assessed on their outcome. Outcome scores are used for research purposes to compare prostheses, surgical techniques, methods of fixation and types of peri-operative care. They are also used for audit to allow comparison between surgeons, departments, institutions and countries. Outcome after surgery can be defined in many different ways such as mortality, morbidity, clinical findings, radiological findings, postoperative complications, rates of re-operation, pain, length of hospital stay and health-related quality-of-life.

Generic short-term clinical outcomes address overall morbidity and mortality related to surgery. Mortality at a variety of time points, the duration of post-operative stay and the incidence of specific complications fall within this group. Morbidity and complications are poorly and inconsistently recorded. ${ }^{1}$ The post-operative morbidity survey ${ }^{2}$ is a recently validated measure of post-operative morbidity ${ }^{3}$ which focuses on indicators of dysfunction of organ systems that can be easily obtained. It can be used to record general complications such as deep-vein thrombosis, pulmonary embolism, wound problems and infection in the immediate post-operative period. It also assesses mobility and can identify orthopaedic-specific complications such as fracture and dislocation.

As the reliability of orthopaedic surgical procedures improves, the assessment of outcome is shifting from the success or failure of a procedure towards patient satisfaction and quality-of-life indicators. Several quality-of- life surveys are now available and they can be divided into three broad categories: generic, disease-specific and joint-specific. Generic surveys assess all facets of health-related qualityof-life whereas disease-specific tools focus on the patient's perceptions of a single disease entity. Joint-specific tools focus on disability relating to a particular joint irrespective of the underlying pathology.

The results from outcome studies can be affected by patient co-morbidity. The physiological and operative severity score for enumeration of mortality and morbidity was developed to address this issue. ${ }^{4}$ A score is allocated to each patient based on the physiological status and the extent of surgery. When comparing patient groups the mean scores must be similar to give meaningful results. An orthopaedic version of this system has now been developed and validated. ${ }^{5}$

\section{Validation of outcome measures}

For an outcome measure to be meaningful, it must be psychometrically evaluated and shown to be reliable, valid and sensitive to change. Tests of reliability answer the question: Is the test measuring something, for example an underlying concept such as mobility, in a reproducible fashion ${ }^{6}$ Internal consistency describes whether a survey measures a single underlying concept and can be tested using Cronbach's alpha. ${ }^{7}$ Reproducibility (or stability) defines whether the questionnaire yields the same results in repeated trials under the same conditions. Paired sets of data can be compared using the kappa coefficient. 
Tests of validity assess whether the survey measures what it is proposed to measure. There are many types of validity: content validity, criterion validity and construct validity. Content validity examines whether items in a questionnaire cover the intended topics. It is a subjective measure and cannot be evaluated statistically. Criterion validity examines how a new measure relates to an established 'gold standard' in the field. It can be measured by the Pearson correlation coefficient between the score for the questionnaire and the 'gold standard'. Construct validity assesses if a single underlying entity is being measured and is assessed using correlation coefficients between scale scores.

Sensitivity to change or responsiveness indicates whether the survey is able to detect clinically significant changes. It is assessed by comparing outcome scores before and after an intervention and is defined as the difference between the mean pre- and post-operative scores divided by the standard deviation of the pre-operative scores.

A detailed description of the development and validation of a new patient-reported outcome score, the Oxford elbow score has been presented in this journal. ${ }^{8}$

\section{Generic quality-of-life outcome measures}

Generic outcome measures aim to assess all dimensions of health-related quality-of-life. The World Health Organisation Quality-of-Life group has recommended that five dimensions should be assessed in any generic quality-of-life survey: physical health, psychological health, social relationship perceptions, function and well-being. ${ }^{9}$ Generic outcome measures are used across a wide range of medical and surgical specialties. Commonly-used measures are the medical outcomes study 36-item short-form health survey (SF-36), ${ }^{10}$ the medical outcomes study 12-item short form health survey (SF-12), ${ }^{11}$ the Nottingham health profile ${ }^{12}$ and the European quality-of-life 5-dimension (EuroQol) questionnaire. ${ }^{13}$ The SF-36 score. This is a 36 -item questionnaire which explores health over the previous four weeks. ${ }^{10}$ Each question has a choice of between two and six answers on a Likert-type scale and each answer is scored between 0 (worst health) and 100 (best health). The questions cover eight health concepts: bodily pain, physical functioning, role limitations due to physical health, general health, mental health, vitality, social functioning and role limitations due to emotional health.

The eight health concepts can be grouped into two higher-order clusters known as the physical component summary (calculated from the bodily pain, physical functioning, role limitations due to physical health and general health scores) and the mental component summary (calculated from the mental health, vitality, social functioning and role limitations due to emotional health scores). The former is most responsive to treatments which alter physical symptoms such as hip replacement. ${ }^{14}$

The SF-36 score was developed in American English but a United Kingdom English version is available. It takes five to ten minutes to complete and is suitable for self- administration or administration by a computer or by an interviewer. Scores are aggregated without standardisation or weighing. The SF-36 score is one of the most evaluated generic questionnaires and is known to be valid and consistent, ${ }^{15,16}$ sensitive ${ }^{14}$ and reproducible. ${ }^{10}$ In patients undergoing total hip replacement, the SF-36 score has been shown to be valid ${ }^{17}$ and reliable. ${ }^{18}$ However, it does have minor 'floor' and 'ceiling' effects. ${ }^{19,20}$ 'Floor' effect refers to data that cannot be less than a defined minimum number. Any deterioration in this group will not be detected. 'Ceiling' effect refers to the opposite situation where data cannot be greater than a defined maximum number. In this group, improvement will not be detected.

The SF-12 score. This is a short form of the SF-36 score and consists of 12 of the 36 questions, but if the sample size is sufficiently large, the SF-12 can produce profiles of the eight SF-36 health concepts. The SF-12 scoring algorithms are weighted and a computer program is available for scoring.

The advantages of the SF-12 over the SF-36 score are that it improves efficiency and lowers cost. The main disadvantage is that it has less construct validity and sensitivity thus producing less precise scores for the eight-scale health profile. ${ }^{11}$

The Nottingham health profile questionnaire. This is a selfadministered questionnaire which takes five to ten minutes to complete. It was developed in English and consists of two parts. Part I contains 38 'yes/no' items covering six dimensions: pain, physical mobility, emotional reactions, energy, social isolation and sleep. Part II has seven 'yes/no' questions concerning problems of daily living. It has been shown to be internally consistent, valid, reproducible and sensitive. ${ }^{21}$ No psychometric analysis has been performed on patients undergoing hip replacement.

The Nottingham health profile has one major disadvantage when compared with the SF-36 score concerning the dichotomous 'yes/no' response format. This restricted response format explores only ill health whereas the SF-36 score with its multiple-response options can detect positive as well as negative states of health. This produces higher ceiling effects in all dimensions for the Nottingham health profile when compared with the SF-36 score. ${ }^{22}$ They both have equal minor floor effects.

The EuroQol questionnaire. This is self-administered and takes approximately five minutes to complete. The first part contains 15 questions which explore five health dimensions: mobility, self care, usual activities, pain and depression. The three possible replies are: 'no problem', 'moderate problem' or 'extreme problem', The second part examines the patient's perception of their overall health and contains a 100-point visual analogue scale.

The questionnaire is known to be both valid ${ }^{23}$ and reliable, ${ }^{24}$ but EuroQol suffers from the same ceiling effects as the Nottingham health profile because of the restricted response format. In patients undergoing hip replacement test-retest reliability has been shown ${ }^{25}$ and there is evidence of construct validity and responsiveness. ${ }^{26}$ 
Disease-specific quality-of-life outcome measures

These provide patient-centred information about a particular disease. This allows comparison of different surgical and medical treatment options for that disease. Qualityof-life outcome measures commonly used to assess arthritis of the hip are the Western Ontario and McMaster Universities (WOMAC) osteoarthritis index ${ }^{27}$ and the arthritis impact measurement scales. ${ }^{28}$ Although these measures are specific for arthritis, they can be used to assess any joint and any intervention.

The WOMAC osteoarthritis index. This was developed in Canadian English for patients with osteoarthritis. ${ }^{27}$ The original version has undergone several refinements and WOMAC 3.1 has been the standard form for several years. It is self-administered and contains 24 questions covering three dimensions: pain, stiffness and physical function. The standard version uses a 48-hour timeframe. The WOMAC index is available in a five-point Likert, 100 $\mathrm{mm}$ visual analogue and 11-point numerical rating format. It is valid, reliable, responsive, easy to complete and simple to score. ${ }^{27}$ Most clinical studies use the Likert and visual analogue versions of WOMAC 3.1. The index has been extensively evaluated in patients undergoing hip replacement and has been shown to be responsive, ${ }^{29}$ have high internal consistency ${ }^{30}$ and acceptable test-retest reliability. $^{31}$

The arthritis impact measurement scale. This was developed in American English to measure outcome in patients with rheumatic disease. It has since been shown to be sensitive in patients with osteoarthritis. The arthritis impact measurement scale $2^{32}$ is a revised version and consists of 78 questions covering 12 scales: mobility, walking and bending, hand and finger function, arm function, self-care tasks, household tasks, social activity, support from family and friends, arthritis pain, work, level of tension and mood. The scores of each scale are normalised to give a value from 0 (good health) to 10 (poor health). This is self-administered and takes approximately 20 minutes to complete. It has been shown to be both valid and reliable. ${ }^{33}$

A further version of the arthritis impact measurement scale has been produced specifically for patients undergoing hip replacement. ${ }^{34}$ This questionnaire consists of 57 items which are scored and weighted to produce four subscales: physiological function, self concept, role function and interdependence. Responsiveness, content and construct validity have been proved for this version of the system. ${ }^{34}$

\section{Hip-specific outcome measures}

The outcome of total hip replacement (THR) was initially assessed by the surgeon using tools such as the Harris hip score (HHS) $)^{35}$ and Charnley score. ${ }^{36}$ Patients and surgeons often differ in their priorities and concerns ${ }^{37}$ and therefore hip-specific quality-of-life surveys were developed to elicit the patient's perception of the outcome of surgery. Such surveys in common use today include the
Oxford hip score (OHS) ${ }^{38}$ and the hip disability and osteoarthritis outcome score. ${ }^{39}$

The HHS, Charnley score and the OHS were all developed to assess patients undergoing THR, irrespective of the underlying diagnosis. The hip disability and osteoarthritis outcome score can be used to assess any intervention on any hip pathology.

Harris hip score. This was developed in English in 1969. The assessment is performed by a surgeon and consists of eight questions and a physical examination. The questions are grouped into three categories: pain, function and level of activity, and the physical examination involves assessing the range of movement of the hip. Scores from each section are simply added together to make a maximum possible score of 100 (indicating the best possible outcome).

The original surgeon-reported measure was modified to create a patient-reported measure. This contains seven items: pain, support for walking, limping, walking distance, climbing stairs, putting on shoes and socks and sitting. Each item is reported using a Likert-type scale with between three and seven possible responses. This version of the HHS gives an overall score of 0 to 100 in which a lower score represents better health status. It has been found to be both valid and reliable in the assessment of the outcome of THR. ${ }^{40}$

Charnley score. This was devised in 1972 and grades hip pain, mobility and walking on a six-point scale. The assessment is performed by the surgeon and lower scores indicate greater disability. The assessment is simple to perform but reflects the opinions of both the surgeon and the patient. There is no psychometric testing of the Charnley score supporting its use.

The Oxford hip score. This was developed in English to assess disability in patients undergoing THR. The survey takes approximately five minutes to complete and assesses health over the previous four weeks. The measure contains 12 questions which assess pain and functional ability and each question has five Likert-type response choices. The overall score ranges from 12 to 60 with a higher score indicating greater disability.

The OHS has been used extensively in orthopaedics and is highly sensitive for patients undergoing primary ${ }^{41}$ and revision THR. ${ }^{42}$ It is internally consistent, reproducible and valid. ${ }^{43}$

The hip disability and osteoarthritis outcome score. This is a 40-item questionnaire based on the knee disability and osteoarthritis outcome score. The questionnaire is selfadministered and takes eight to ten minutes to complete. Each question has five possible answers which are scored from 0 to 4 . The questions are grouped into five subscales: pain, other symptoms, activities of daily living, sport and hip-related quality of life. The score for each subscale is simply the sum of the individual question scores. The hip disability and osteoarthritis outcome score is then transformed on to a scale from 0 to 100 with 100 indicating the best possible outcome. 
Table I. Criteria for a positive post-operative morbidity survey score (reproduced with permission from Elsevier ${ }^{3}$ )

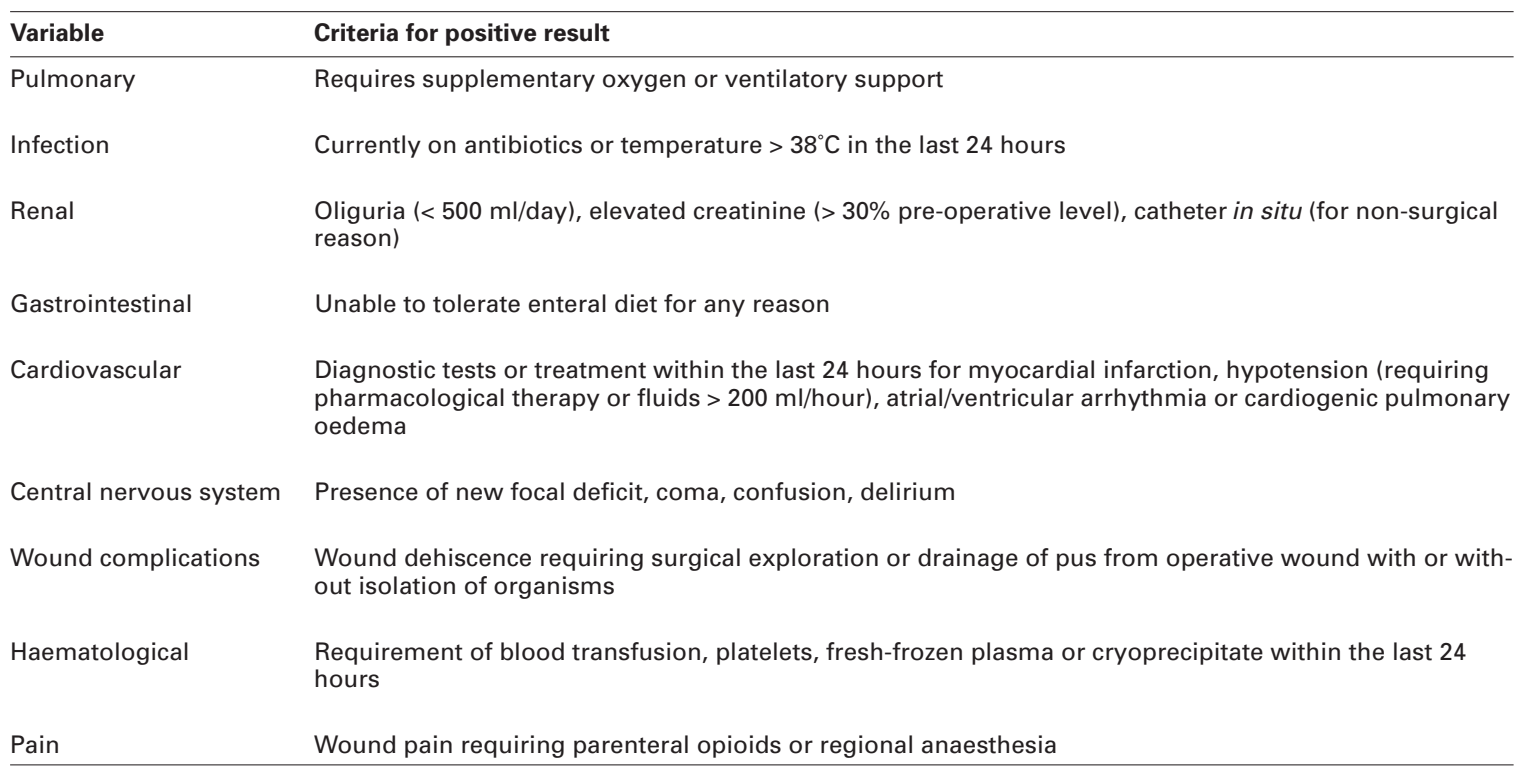

This scoring system contains all the WOMAC Likert 3.0 questions in an identical form and therefore can be used to calculate the WOMAC scores. It has been shown to be both valid and responsive. ${ }^{39}$

\section{Short-term clinical outcome measures}

The short-term clinical impact of operations and the associated physiological disturbance can be described in different ways. Although mortality is important it is now so infrequent for most types of surgery that it is not useful as a comparative index. Length of hospital stay has been used as a marker of clinical outcome, but is recognised to be a compound measure of clinical status and the way in which a hospital functions. ${ }^{44}$

The post-operative morbidity survey ${ }^{1}$ is a measure of postoperative morbidity which focuses on indicators of dysfunction of organ systems that are easy to collect. Data for the post-operative morbidity survey (Table I) are collected from in-patients on selected post-operative days using observation charts, medication charts, patient notes, routine blood test results and direct patient questioning and observation. It requires no additional investigations. It can be used to assess short-term morbidity after any type of surgery and has been used in outcomes research ${ }^{45}$ and in effectiveness research. ${ }^{46} \mathrm{It}$ has been shown to be reliable, valid and acceptable to patients. $^{3}$

The post-operative morbidity survey identifies morbidity which is sufficient to delay discharge from hospital. Therefore, as well as providing useful research and audit data, it can assist with the planning of discharge and contribute to cost reduction.

\section{Conclusions}

Orthopaedic interventions are assessed on their outcome. There are several ways of assessing outcome including short-term post-operative measures and long-term patientcentred quality-of-life outcome measures. Since short-term post-operative surveys are only now evolving, it remains to be seen whether they will show any correlation with longterm outcome questionnaires. It is, therefore, advisable to use a combination of both measures to assess any operative intervention.

There are three main categories of long-term quality-oflife outcome measures, namely, generic, disease-specific and joint-specific. The most psychometrically evaluated generic measure is the SF-36 score, the most evaluated diseasespecific measure is the WOMAC index and the most evaluated hip-specific measure is the OHS. Any hip intervention should be assessed by both a generic and specific outcome measure. They examine different aspects of the patient's function and together provide more information than one questionnaire alone.

The choice of which generic and specific questionnaire to use depends on several factors including the aim of the study, the level of observation required, the funding available and the context of the study. Long-term follow-up is required, and therefore simple, short questionnaires may make long-term studies more feasible.

\section{References}

1. Bruce J, Russell EM, Mollison J, Krukowski ZH. The measurement and monitoring of surgical adverse events. Health Technol Assess 2001;5:1-194.

2. Bennett-Guerrero E, Welsby I, Dunn TJ, et al. The use of a postoperative morbidity survey to evaluate patients with prolonged hospitalization after routine, moderaterisk, elective surgery. Anesth Analg 1999;89:514-19.

3. Grocott MP, Browne JP, Van der Meulen J, et al. The post-operative morbidity survey was validated and used to describe morbidity after major surgery. J Clin Epidemiol 2007;60:919-28.

4. Copeland GP, Jones D, Walters M. POSSUM: a scoring system for surgical audit. Br J Surg 1991;78:355-60.

5. Mohamed K, Copeland GP, Boot DA, et al. An assessment of the POSSUM system in orthopaedic surgery. J Bone Joint Surg [Br] 2002;84-B:735-9. 
6. Streiner DL. Being inconsistent about consistency: when coefficient alpha does and doesn't matter. J Personality Assessment 2003;80:217-22.

7. Cronbach LJ. Coefficient alpha and the internal structure of tests. Psychometrika 1951;16:297-334.

8. Dawson J, Doll H, Boller I, et al. The development and validation of a patient-reported questionnaire to assess outcomes of elbow surgery. J Bone Joint Surg [Br]2008;90-B:46673.

9. No authors listed. Study Protocol of the World Health Organization project to develop a quality of life instrument (WHOOOL). Qual Life Res 1993;2:153-9.

10. Ware JE Jr, Sherbourne CD. The MOS 36-item short-form health survey (SF-36). I: conceptual framework and item selection. Med Care 1992;30:473-83.

11. Ware J Jr, Kosinski M, Keller SD. A 12-item short-form health survey: construction of scales and preliminary tests of reliability and validity. Med Care 1996;34:220-33

12. Hunt SM, McEwan J. The development of a subjective health indicator. Sociol Health IIIn 1980;2:231-46.

13. Brooks R. EuroQol: the current stage of play. Health policy 1996;37:53-72.

14. Kantz JN, Larson MG, Philips CB, Fossel AH, Liang MH. Comparative measurement sensitivity of shorter and longer health status instruments. Med Care 1992;30:917-25

15. Brazier JE, Harper R, Jones NM, et al. Validating the SF-36 health survey questionnaire: new outcome measure for primary care. BMJ 1992;305:160-4

16. McHorney CA, Ware JE, Raczek AE. The MOS 36-item short form health survey (SF36): II. psychometric and clinical tests of validity in measuring physical and mental health constructs. Med Care 1993;31:247-63.

17. Feeny D, Wu L, Eng K. Comparing short form $6 D$, standard gamble, and Health Utilities Index Mark 2 and Mark 3 utility scores: results from total hip arthroplasty patients. Qual Life Res 2004;13:1659-70.

18. Mahomed NN, Arndt DC, McGrory BJ, Harris WH. The Harris hip score: comparison of patient self-report with surgeon assessment. J Arthroplasty 2001;16:575-80.

19. Shields RK, Enloe LJ, Leo KC. Health related quality of life in patients with total hip or knee replacement. Arch Phys Med Rehabil 1999;80:572-9.

20. Mangione CM, Goldman L, Orav EJ, et al. Health-related quality of life after elective surgery: measurement of longitudinal changes. J Gen Intern Med 1997;12:686-97.

21. Jenkinson C, Fitzpatrick R, Argyle M. The Nottingham Health Profile: an analysis of its sensitivity in differentiating illness groups. Soc Sci Med 1988;27:1411-14.

22. Beaton DE, Bombardier C, Hogg-Johnson SA. Measuring health in injured workers: a cross-sectional comparison of five generic health status instruments in workers with musculo-skeletal injuries. Am J Ind Med 1996;29:618-31.

23. Brazier $\mathbf{J}$, Jones $\mathbf{N}$, Kind $\mathbf{P}$. Testing the validity of the EuroQol and comparing it with the SF-36 health survey questionnaire. Qual Life Res 1993;2:169-80.

24. Van Agt HM, Essink-Bot ML, Krabbe PF, Bonsel GJ. Test-retest reliability of health state valuations collected with the EuroOol questionnaire. Soc Sci Med 1994;39:1537-44.

25. Luo N, Chew LH, Fong KY, et al. Validity and reliability of the EQ-5D self-report questionnaire in English-speaking Asian patients with rheumatic diseases in Singapore. Qual Life Res 2003;12:87-92

26. Tidermark J, Bergström G, Svensson 0, Törnkvist H, Ponzer S. Responsiveness of the Euroqol (EQ 5-D) and the SF-36 in elderly patients with displaced femoral neck fractures. Qual Life Res 2003;12:1069-79.

27. Bellamy N, Buchanan WW, Goldsmith CH, Campbell J, Stitt L. Validation study of WOMAC: a health status instrument for measuring clinically-important patient-relevant outcomes following total hip or knee arthroplasty in osteoarthritis. J Orthopaedic Rheumatol 1988;1:95-108.
28. Meenan RF, Gertman PM, Mason JH. Measuring health status in arthritis: the arthritis impact measurement scales. Arthritis Rheum 1980;23:146-52.

29. Bachmeier CJ, March LM, Cross MJ, et al. A comparison of outcomes in osteoarthritis patients undergoing total hip and knee replacement surgery. Osteoarthritis Cartilage 2001;9:137-46.

30. Kennedy D, Stratford PW, Pagura SMC, et al. Exploring the factorial validity and clinical interpretability of the Western Ontario and McMaster Universities Osteoarthritis Index (WOMAC). Physiotherapy Canada 2003;55:160-8.

31. Stucki G, Sangha 0, Stucki S, et al. Comparison of the WOMAC (Western Ontario and McMaster Universities) osteoarthritis index and a self-report format of the selfadministered Lequesne-Algofunctional index in patients with knee and hip osteoarthritis. Osteoarthritis Cartilage 1998;6:79-86.

32. Meenan RF, Mason JH, Anderson JJ, Guccione AA, Kazis LE. AIMS2: the content and properties of a revised and expanded Arthritis Impact Measurement Scales Health Status Questionnaire. Arthritis Rheum 1992;35:1-10.

33. Lorig K, Chastain RL, Ung E, Shoor S, Holman HR. Development and evaluation of a scale to measure perceived self-efficacy in people with arthritis. Arthritis Rheum 1989:32:37-44.

34. Selman SW. Impact of total hip replacement on quality of life. Orthop Nurs 1989;8:43-9.

35. Harris WH. Traumatic arthritis of the hip after dislocation and acetabular fractures: treatment by mold arthroplasty: an end-result study using a new method of result evaluation. J Bone Joint Surg [Am] 1969;51-A:737-55.

36. Charnley J. The long-term results of low-friction arthroplasty of the hip performed as a primary intervention. J Bone Joint Surg [Br] 1972;54-B:61-76.

37. Wright JG, Rudicel S, Feinstein AR. Ask patients what they want: evaluation of individual complaints before total hip replacement. J Bone Joint Surg [Br] 1994;76B:229-34.

38. Dawson J, Fitzpatrick R, Carr A, Murray D. Questionnaire on the perceptions of patients about total hip replacement. J Bone Joint [Br] 1996;78-B:185-90.

39. Nilsdotter AK, Lohmander LS, Klässbo M, Roos EM. Hip disability and osteoarthritis outcome score (HOOS): validity and responsiveness in total hip replacement. BMC Musculoskeletal Disord 2003;4:10.

40. Söderman P, Malchau H. Is the Harris hip score system useful to study the outcome of total hip replacement? Clin Orthop 2001;384:189-97.

41. Ostendorf M, Van Stel HF, Buskens E, et al. Patient-reported outcome in total hip replacement: a comparison of five instruments of health status. J Bone Joint Surg [Br] 2004;86-B:801-8.

42. Dawson J, Fitzpatrick R, Frost S, et al. Evidence for the validity of a patient-based instrument for the assessment of outcome after revision hip replacement. J Bone Joint Surg [Br] 2001;83-B:1125-9.

43. Dawson J, Fitzpatrick R, Murray D, Carr A. Comparison of measures to assess outcomes in total hip replacement surgery. Qual Health Care 1996;5:81-8.

44. Daley J, Henderson WG, Khuri SF. Risk-adjusted surgical outcomes. Annu Rev Med 2001;52:275-87.

45. Bennett-Guerrero E, Feierman DE, Barclay GR, et al. Preoperative and intraoperative predictors of postoperative morbidity, poor graft function, and early rejection in 190 patients undergoing liver transplantation. Arch Surg 2001;136:1177-83.

46. Wakeling HG, McFall MR, Jenkins CS, et al. Intraoperative oesophageal Doppler guided fluid management shortens postoperative hospital stay after major bowel surgery. Br J Anaesth 2005;95:634-42. 